\title{
Influence of Oxidative Stress Markers on Coronary No-reflow After Primary Percutaneous Coronary Intervention for Patients with Acute Myocardial Infarction
}

\author{
Mahmoud Ragab Darwish ${ }^{1, *}$, Walaa Farid², Ahmed Mokhtar El-Kersh ${ }^{3}$ \\ ${ }^{1}$ Cardiology Department, Health Insurance, Tanta, Egypt \\ ${ }^{2}$ Cardiology Department, Menoufia University, Shebin Elkom, Egypt \\ ${ }^{3}$ Cardiology Department, Faculty of Medicine, Menofia University, Shebin Elkom, Egypt \\ Email address: \\ mahmoudragabdarwish75@gmail.com (M. R. Darwish) \\ ${ }^{*}$ Corresponding author
}

\section{To cite this article:}

Mahmoud Ragab Darwish, Walaa Farid, Ahmed Mokhtar El-Kersh. Influence of Oxidative Stress Markers on Coronary No-reflow After Primary Percutaneous Coronary Intervention for Patients with Acute Myocardial Infarction. Cardiology and Cardiovascular Research. Vol. 5, No. 1, 2021, pp. 16-24. doi: 10.11648/j.ccr.20210501.13

Received: December 20, 2020; Accepted: December 30, 2020; Published: January 15, 2021

\begin{abstract}
Background: ST-elevation myocardial infarction (STEMI) is one of the leading causes of mortality and morbidity worldwide. However, survival after acute STEMI has considerably improved due to increasing symptom recognition, accurate diagnosis and effective timely reperfusion. This study aimed to investigate the relation between the level of oxidative stress markers and coronary no-reflow after primary percutaneous coronary intervention for patients with acute myocardial infarction. Patients \& Methods: This prospective cohort study included 90 patients admitted with acute STEMI at cardiovascular medicine department Naser Institute hospital, during the period from June 2018 till 12 months. Patients were divided into 2 groups according to the post primary PCI thrombolysis in myocardial infarction (TIMI) flow score into: Group I: 45 patients with noreflow phenomenon. Group II: 45 patients with TIMI flow $\geq 2$ after primary PCI. They all underwent primary PCI within 24 hours of presentation. Results: This study showed increased concentrations of Malondialdehide (MDA) in the circulation of patients with no-reflow indicating increased lipid peroxidation which could be attributed to a deficiency of antioxidant defense mechanism. In group I, pt with coronary no-reflow MDA level ranged from $2.8-4.5 \mathrm{nmol} / \mathrm{mL}$ with mean $3.9 \pm 1.5 \mathrm{nmol} / \mathrm{mL}$, while in group II control group, MDA level ranged from $1.1-2.1 \mathrm{nmol} / \mathrm{mL}$ with mean $1.55 \pm 0.4 \mathrm{nmol} / \mathrm{mL}$, there was statistically significant difference between the two groups $(\mathrm{P}$ value $<0.004)$. Conclusions: no-reflow phenomenon after primary PCI can be predicted using the oxidative stress markers.
\end{abstract}

Keywords: Oxidative Stress, Percutaneous Coronary Intervention, St-elevation Myocardial Infarction

\section{Introduction}

Timely reperfusion of the infarct-related coronary artery using percutaneous coronary intervention (PCI) is the optimum ST segment elevation myocardial infarction (STEMI) treatment, reducing infarct size, minimizing myocardial damage, preserving ventricular function, and decreasing morbidity and mortality. Yet, despite opening the affected epicardial coronary artery, myocardial perfusion may sometimes not be restored, even resulting in no-reflow $[1,2]$ The no-reflow phenomenon is a serious complication and an independent risk factor for worse clinical adverse outcome.
No-reflow is a multifactorial and complex phenomenon that causes perfusion deficits in the microvasculature [3]. The noreflow phenomenon is defined as the inability to reperfuse regions of the myocardium despite removal of a large epicardial coronary artery occlusion [4]. The patients with no-reflow exhibit a higher prevalence of; earlyost infarction complications; left adverse ventricular remodeling; late repeat hospital stays for heart failure; and mortality [5]. Ischemic and reperfusion injuries are important pathogenetic mechanisms of no-reflow following ST elevation myocardial infarction (STEMI). Ischemia results in impaired antioxidant defense and subsequent reperfusion results in an increased 
concentration of reactive oxygen species (ROS) [6, 7]. Highdensity lipoprotein (HDL) cholesterol exerts cardio protective properties through its antioxidant activity and antiinflammatory effects [8].

Antioxidants, including various agents such as enzymes (glutathione peroxidase, superoxide dismutase, and catalase), large molecules (albumin and ferritin), and small molecules (uric acid, glutathione, bilirubin, vitamin $\mathrm{C}$, and vitamin E), play an important role in the cellular protection cascade against oxidative damage [9]. The total antioxidant status (TAS) mirrors the activity potential of the antioxidant system. Several methods have been introduced to measure the total antioxidant capacity (TAC) in different biological specimens [10]. The measurement of TAC reflects the antioxidative status of plasma because antioxidative effects of the plasma antioxidant components are additive [11].

The assessment of plasma TAC can be more useful than the measurement of individual antioxidant levels in cells and plasma because it could determine the synergistic interaction among different individual antioxidants $[12,13]$. This study aimed to investigate the relation between the level of oxidative stress markers and coronary no-reflow after primary percutaneous coronary intervention for patients with acute myocardial infarction.

\section{Patients and Methods}

A prospective study was conducted on 90 patients their ages ranged from 38 to 74 years (69 males and 21 females) admitted with acute STEMI and treated with primary PCI within 24 hours of presentation at cardiovascular medicine department Naser Institute hospital, during the period from June 2018 till 12 months. Patients were divided into 2 groups according to the post primary PCI thrombolysis in myocardial infarction (TIMI) flow score into: Group I: (Case group) 45 patients with no-reflow phenomenon, patients with thrombolysis in myocardial infarction (TIMI) $0-1$ flow post primary PCI, in the absence of dissection, thrombus, spasm or high-grade residual stenosis, were considered as no-reflow case. Group II: (Control group) 45 patients two consecutive STEMI patients after each case, with TIMI flow $\geq 2$ after primary PCI. Inclusion criteria: Patients presenting with STEMI and treated with primary PCI within 24 hours of presentation. Criteria of exclusion: Patients with acute coronary syndrome other than STEMI, coronary artery dissection, coronary artery spasm, high-grade residual coronary stenosis, previous myocardial infarction, previous PCI, previous CABG.

After having written consent from included patients in the study. All patients were subjected to Demographic data taking, Complete clinical examination, Hypertension was defined as office systolic blood pressure (SBP) values $\geq 140$ $\mathrm{mmHg}$ and/or diastolic BP (DBP) values $\geq 90 \mathrm{mmHg}{ }^{128}$.

Dyslipidemia is defined as serum total cholesterol level over $200 \mathrm{mg} / \mathrm{dl}$ or triglycerides more than $150 \mathrm{mg} / \mathrm{dl}$ or current treatment with lipid lowering medication [14]. Patients who had previous history or current diagnosis of DM.
DM is defined by an elevated level of blood glucose. The classification of DM is based on recommendations from the World Health Organization (WHO) and the American Diabetes Association (ADA).

Standard 12-lead ECG was obtained within 10 minutes of first medical contact (FMC) according to ESC guidelines 2017 including: (limb leads I, II, III, aVR, aVL, aVF, and Chest leads fromV1 to V6) for all patients on admission to the hospital. Right pericardial leads (V3R, V4R, V5R, V6R) and posterior chest leads (V7 to V9) were done for some patients to detect posterior wall and right ventricular infarction.

\subsection{Pre-procedural Medications:}

All patients received a loading dose of Aspirin $300 \mathrm{mg}$ and clopidogrel $600 \mathrm{mg}$ or ticagrelor $180 \mathrm{mg}$. Intravenous administration of Unfractionated Heparin with a dose of 70-100 U/kg was given when no glycoprotein (GP) IIb/IIIa inhibitor was used and 50-60 U/kg with the use of GP IIB/IIIa inhibitor. In patients who were treated with GP $\mathrm{IIb} / \mathrm{III}$ inhibitors (Eptifibatide or Tirofiban), the agent was administrated before the primary PCI procedure in the coronary care unit, as was the continuous infusion after the procedure according to operator point of view. The decision to administer glycoprotein IIb/IIIa inhibitors was made by the interventional cardiologist. Visualization of coronary arteries lumen was done using intravascular injection of non-ionic contrast medium through the used catheter into the engaged coronary artery. Grade of blood flow before procedure was determined by TIMI blood flow grade classification system.

Angiographic stenosis was defined as a luminal diameter reduction of $\geq 50 \%$ by quantitative coronary angiography, and critical stenosis was defined as $\geq 70 \%$ narrowing of the coronary artery luminal diameter. Complete coronary occlusion was defined as absence of antegrade flow of contrast media beyond a specific vascular segment. The infarct-related lesion was determined by the typical vascular morphological features of thrombus-laden or hazy filling defects along with compromise of distal flow.

IRA was identified according to the culprit lesion on the basis of the infarct location on the admission ECG and the angiographic findings (target vessel, lesion characteristics). Multivessel disease was defined as presence of $\geq 1$ lesion with $>50 \%$ stenosis in $\geq 1$ major epicardial coronary artery or its major branches remote from the IRA.

To evaluate clot burden, Thrombus grading score was used: TIMI thrombus grade $0:$ no cine-angiographic characteristics of thrombus were present.

TIMI thrombus grade 1: possible thrombus was present with such angiographic characteristics as decreased contrast density, haziness, irregular lesion contour, or a smooth convex "meniscus" at the site of total occlusion suggestive but not diagnostic of thrombus.

TIMI thrombus grade 2: there was definite thrombus, with the largest dimensions $\leq 1 / 2$ the vessel diameter. TIMI thrombus grade 3: there was definite thrombus but with the 
largest linear dimension $>1 / 2$ but $<2$ vessel diameters. TIMI thrombus grade 4: there was definite thrombus, with the largest dimension $\geq 2$ vessel diameters. TIMI thrombus grade 5: the ischemic vessel was totally occluded with TIMI 0 flow.

PCI with or without stenting was immediately performed with a 6-Fr guiding catheter. Thrombus aspiration, balloon pre-dilatation and post- dilatation were performed when indicated. The choice of stents (bare metal stent or drugeluting stent) was left to the operator's discretion. Reperfusion success is measured by TIMI blood flow grade: Reperfusion was considered successful (TIMI 3) or abnormal (TIMI 0-1-2) according to the TIMI blood flow grade. Patients with (TIMI) $0-1$ flow post primary PCI, in the absence of dissection, thrombus, spasm or high-grade residual stenosis, are considered as no-reflow cases.

\subsection{Echocardiography}

All studies were performed using (a GE vivid seven Cardiac ultrasound phased array system with tissue Doppler imaging using M4S transducer 4M. Hz.). Two- Dimensional echocardiographic assessment by M-mode and modified Simpson method were done during admission after PPCI. 2D Echocardiography was done in partial left lateral decubitus position to: Assess LV systolic function using Simpson's method in the apical $4 \&$ apical 2 views also we assessed Left ventricular volumes. (End diastolic volume EDV and end systolic volume ESV).

Assess Segmental wall motion abnormalities and global wall motion A 17 segment model for LV segmentation was used to evaluate regional wall motion abnormalities. The wall motion of each segment was scored from 1 (normal) to 4 (dyskinetic).

\subsection{Statistical Analysis}

The analysis was calculated by SPSS software package version 25 . The qualitative parameters were described by number of frequency and percentage while the quantitative variables were described by mean, standard deviation and range. Normality of qualitative variables was tested by Kolmogorov-Smirno test. The comparison of independent quantitative variables was calculated by $\mathrm{T}$ independent test. The comparison between two qualitative variables was done by Chi square, Fisher's exact fisher and Monte Carlo tests. The risk estimate was evaluated by odds ratio with $95 \%$ confidence interval.

\section{Results}

The present study was conducted on 90 patients included in the study, 69 patients of the study population were males and 21 were females. The age of the study population ranged from 38 to 74 years. In group I, included 41 males $(91.1 \%)$ and 4 females $(8.9 \%)$. the age of the patients ranged from 59 - 74 years. There were 43 patients $<60$ years $(95.6 \%)$ and only two patient was $>60$ years $(4.4 \%)$. In group II, included 27 males $(60 \%)$ and 18 females $(40 \%)$. the age ranged from $38-68$ years. There were 8 patients $<60$ years $(17.8 \%)$ and 37 patients were $>60$ years $(82.8 \%)$. There was statistically significant difference between the two groups (Age $<60$ years, $\mathrm{P}$ value $<0.001)$. There was statistically significant difference between the two groups (if male, $\mathrm{P}$ value $=0.001$ ). Table 1

Table 1. Demographic data of cases and control groups.

\begin{tabular}{llllll}
\hline Variables & Case (no-reflow) $(\mathbf{n}=\mathbf{4 5})$ & Control $(\mathbf{n = 4 5})$ & & Sig. test \\
\hline Age & & & & & \\
$<60$ & 2 & $4.4 \%$ & 37 & $82.2 \%$ & 55.340 \\
$>60$ & 43 & $95.6 \%$ & 8 & $17.8 \%$ & \\
Sex & & & & & \\
Male & 41 & $91.1 \%$ & 27 & $60.0 \%$ & 11.791 \\
Female & 4 & $8.9 \%$ & 18 & $40.0 \%$ & $0.001 *$ \\
\hline
\end{tabular}

* Statisticall Significant

Table 2, showed that 62 patients were diabetics, 60 patients were hypertensive, 47 patients were active smokers, 41 patients were dyslipidemic and 20 patients having family history of previous coronary artery diseases. 61 patients of the study population were diabetic. In group I, 39 patients were diabetics $(84.4 \%)$, while in group II, 24 patients were diabetics $(53.3 \%)$. There was statistically significant difference between the studied groups, $\mathrm{P}$ value $=0.001$ ). 60 patients of the study population were hypertensive. In group I, 39 patients were hypertensive $(86.7 \%)$, while in group II, 21 patients were hypertensive $(46.7 \%)$. There was statistically significant difference between the studied groups $\mathrm{P}$ value $>0.001$ ).
47 patients of the study population were active smokers. In group I, 21 patients were smokers $(46.7 \%)$, while in group II, 26 patients were active smokers $(57.8 \%)$. There was no statistically significant difference between the two groups ( $\mathrm{P}$ value $=0.291$ ) (Table 2).

41 of the study population were known to have dyslipidemia, In group I, 20 patients were dyslipidemic (44.4\%), while in group II, 21 patients were dyslipidmic $(46.7 \%)$ ( $\mathrm{P}$ value=0.832). 20 of the study population were known to have family history of CAD, $26.7 \%$ of group I and $17.8 \%$ of group II. There was no statistical significant difference between the two groups $(\mathrm{P}$ value $=0.310)$. (Table 2) 
Table 2. Risk factors distribution among cases and control groups.

\begin{tabular}{|c|c|c|c|c|c|c|}
\hline \multirow{2}{*}{$\begin{array}{l}\text { Variables } \\
\mathrm{DM}\end{array}$} & \multicolumn{2}{|c|}{ Case (no-reflow) $(n=45)$} & \multicolumn{2}{|c|}{ Control (n=45) } & \multirow[t]{2}{*}{ Sig. test } & \multirow[t]{2}{*}{ P. } \\
\hline & & & & & & \\
\hline No & 7 & $15.6 \%$ & 21 & $46.7 \%$ & 10.161 & $0.001 *$ \\
\hline Yes & 38 & $84.4 \%$ & 24 & $53.3 \%$ & & \\
\hline \multicolumn{7}{|c|}{ Hypertension } \\
\hline No & 6 & $13.3 \%$ & 24 & $53.3 \%$ & 16.200 & $<0.001^{*}$ \\
\hline Yes & 39 & $86.7 \%$ & 21 & $46.7 \%$ & & \\
\hline \multicolumn{7}{|l|}{ Smoking } \\
\hline No & 24 & $53.3 \%$ & 19 & $42.2 \%$ & 1.113 & 0.291 \\
\hline Yes & 21 & $46.7 \%$ & 26 & $57.8 \%$ & & \\
\hline \multicolumn{7}{|c|}{ Dyslipidemia } \\
\hline No & 25 & $55.6 \%$ & 24 & $53.3 \%$ & 0.045 & 0.832 \\
\hline Yes & 20 & $44.4 \%$ & 21 & $46.7 \%$ & & \\
\hline \multicolumn{7}{|c|}{ Family History } \\
\hline No & 33 & $73.3 \%$ & 37 & $82.2 \%$ & 1.029 & 0.310 \\
\hline Yes & 12 & $26.7 \%$ & 8 & $17.8 \%$ & & \\
\hline
\end{tabular}

* Statistically Significant

In this study population, the systolic blood pressure was $>90 \mathrm{mmHg}$ in 32 patients, the other 58 patients were $<90$ $\mathrm{mmHg}$. In group I, the systolic blood pressure was $>90$ $\mathrm{mmHg}$ in 28 patients $(62.2 \%)$, and $<90 \mathrm{mmHg}$ in the other 17 patients $(37.8 \%)$. In group II, the systolic blood pressure was $>90 \mathrm{mmHg}$ in 4 patients $(8.9 \%)$, and $<90 \mathrm{mmHg}$ in the other 41 patients $(91.1 \%)$. There was statistically significant difference between the two groups $(\mathrm{SBP}<90 \mathrm{mmHg}, \mathrm{P}$ value $>0.001$ ). Table 3

In this study population, heart rate was $<75 \mathrm{~b} / \mathrm{min}$ in 71 patients, the other 19 patients were $>75 \mathrm{~b} / \mathrm{min}$. In group I, the heart rate was $<75 \mathrm{~b} / \mathrm{min}$ in 34 patients $(75.6 \%$ ), and $>75$ $\mathrm{b} / \mathrm{min}$ in the other 19 patients $(23.3 \%)$. In group II, the heart rate was $<75 \mathrm{~b} / \mathrm{min}$ in 50 patients $(83.3 \%)$, and $>75 \mathrm{~b} / \mathrm{min}$ in the other 11 patients $(24.4 \%)$. There was no statistically significant difference between the two groups $(\mathrm{P}$ value $=0.438)$ (table 3).

In this study population, the random blood glucose (RBG) was $<300 \mathrm{mg} / \mathrm{dl}$ in 52 patients, the other 38 patients were $>300 \mathrm{mg} / \mathrm{dl}$. In group I, the RBG was $<300 \mathrm{mg} / \mathrm{dl}$ in 38 patients $(84.4 \%)$, and $>300 \mathrm{mg} / \mathrm{dl}$ in the other 7 patients (15.6\%). In group II, the RBG was $<300 \mathrm{mg} / \mathrm{dl}$ in 14 patients $(31.1 \%)$, and $>300 \mathrm{mg} / \mathrm{dl}$ in the other 31 patients $(68.9 \%)$. There was statistically significant difference between the two groups $(\mathrm{RBG}>300 \mathrm{mg} / \mathrm{dl}, \quad \mathrm{P}$ value $>0.001)$ (table 3). 32 patients of the study population presented with Killip class $\geq$ 3 while 58 patients presented with Killip class $>3$. In group I, 28 patients presented with Killip class $\geq 3(62.2 \%)$, the other 17 patients presented with Killip class $>3$ (37.8\%). In group II, 4 patients presented with Killip class $\geq 3(8.9 \%)$, the other 41 patients presented with Killip class $>3(91.1 \%)$. There was statistically significant difference between the studied groups (Killip $\geq 3$, P value $>0.001$ ) (table 3 ).

44 patients of the study population presented with anterior STEMI while 21 patients presented with inferior STEMI, 21 patients presented with lateral STEMI and 4 patients presented with STEMI other than the mentioned before. In group I, 32 patients presented with anterior STEMI (71.1\%),
13 patients presented with inferior STEMI (28.9\%) and no patients presented with lateral STEMI nor other sites. In group II, 12 patients presented with anterior STEMI (26.7\%), 8 patients presented with inferior STEMI (17.8\%), 21 patients presented by lateral STEMI (46.7\%) and 4 patients presented with STEMI sites other than the previously mentioned $(8.9 \%)$. There was statistically significant difference between the studied groups (Anterior MI, P value $>0.001$ ). (Table 3 ). Ischemic time was $<6$ hours in 60 patients of the study population while the other 30 patients came before 6 hours of duration since the onset of symptoms. In group I, Ischemia time in all of them was $<6$ hours $(100 \%)$. In group II, Ischemic time was $<6$ hours in 15 patients $(33.3 \%)$ and $>6$ hours in the other 30 patients $(66.7 \%)$. There was statistically significant difference between the studied groups (P value $>0.001$ ). (Table 3 )

Infarct related artery (IRA): in 55 of the study population, the left anterior descending artery (LAD) was the IRA, while in 18 patients the left circumflex coronary artery (LCX) was the IRA and in 17 cases it was the right coronary artery (RCA) table (4). In group I, the IRA was the LAD in 34 patients $(75.6 \%))$, the LCX in 3 patients $(6.7 \%)$ and the RCA in 8 patients $(17.8 \%)$. In group II, the IRA was the LAD in 21 patients $(46.7 \%)$, the LCX in 15 patients $(33.3 \%)$ and the RCA in 9 patients $(20 \%)$. There was statistically significant difference between the two groups regarding the IRA (P value $=0.004$ ).

In 44 patients of the study population, the lesion was at the proximal segment of the IRA, while in 34 patients the lesion was at the midsegment and in 13 cases it was distal (table 4). In group I, the lesion was proximal in 32 patients $(71.1 \%)$, at midsegment in 13 patients $(28.9 \%)$ and there were no cases having the lesion distally. In group II, the lesion was proximal in 12 patients $(26.7 \%)$, at midsegment in 20 patients $(44.4 \%)$ and distally in 13 patients $(28.9 \%)$. There was statistically significant difference between the two groups ( $\mathrm{P}$ value $>0.001)$. 
Table 3. Clinical assessment of cases and control groups.

\begin{tabular}{|c|c|c|c|c|c|c|}
\hline \multirow{2}{*}{\begin{tabular}{|l} 
Variables \\
Systolic Blood Pressure
\end{tabular}} & \multicolumn{2}{|c|}{ Case (no-reflow) $(n=45)$} & \multicolumn{2}{|c|}{ Control $(n=45)$} & \multirow[t]{2}{*}{ Sig. test } & \multirow[t]{2}{*}{$\mathbf{P}$. } \\
\hline & & & & & & \\
\hline$>90$ & 17 & $37.8 \%$ & 41 & $91.1 \%$ & 27.931 & $<0.001 *$ \\
\hline$<90$ & 28 & $62.2 \%$ & 4 & $8.9 \%$ & & \\
\hline \multicolumn{7}{|l|}{ Heart Rate } \\
\hline$<75$ & 11 & $24.4 \%$ & 8 & $17.8 \%$ & \multirow[t]{2}{*}{0.600} & \multirow[t]{2}{*}{0.438} \\
\hline$>75$ & 34 & $75.6 \%$ & 37 & $82.2 \%$ & & \\
\hline \multicolumn{7}{|c|}{ RBS at Presentation } \\
\hline$<300$ & 7 & $15.6 \%$ & 31 & $68.9 \%$ & \multirow[t]{2}{*}{26.235} & \multirow[t]{2}{*}{$<0.001^{*}$} \\
\hline$>300$ & 38 & $84.4 \%$ & 14 & $31.1 \%$ & & \\
\hline \multicolumn{7}{|c|}{ KILLIP Class } \\
\hline$<$ III & 17 & $37.8 \%$ & 41 & $91.1 \%$ & \multirow[t]{3}{*}{27.931} & \multirow[t]{2}{*}{$<0.001^{*}$} \\
\hline$\geq \mathrm{III}$ & 28 & $62.2 \%$ & 4 & $8.9 \%$ & & \\
\hline \multicolumn{6}{|c|}{ Location of Infarction } & \\
\hline Anterior & 32 & $71.1 \%$ & 12 & $26.7 \%$ & \multirow{4}{*}{35.281} & \multirow{4}{*}{$<0.001^{*}$} \\
\hline Inferior & 13 & $28.9 \%$ & 8 & $17.8 \%$ & & \\
\hline Lateral & 0 & $0.0 \%$ & 21 & $46.7 \%$ & & \\
\hline Other & 0 & $0.0 \%$ & 4 & $8.9 \%$ & & \\
\hline \multicolumn{7}{|c|}{ Total Ischemia Time } \\
\hline$<6$ hours & 0 & $0.0 \%$ & 30 & $66.7 \%$ & \multirow[t]{2}{*}{45.000} & \multirow[t]{2}{*}{$<0.001^{*}$} \\
\hline$>6$ hours & 45 & $100.0 \%$ & 15 & $33.3 \%$ & & \\
\hline
\end{tabular}

* Statistically Significant

Number of diseased vessels; In group I, 17 patients had multi-vessel disease $(37.8 \%)$. In group II, 2 patients had multi vessel diseases $(4.4 \%)$. There was statistically significant difference between the two groups (P value $>0.001$ ). (table 4)

\subsection{Pre-procedural TIMI Flow Score}

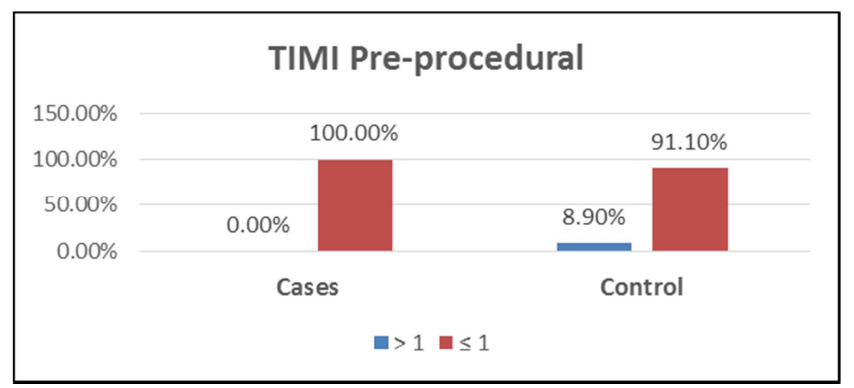

Figure 1. Pre-procedural TIMI flow score in both groups.

86 patients of the study population had TIMI flow $>2$, while 4 patients had TIMI flow $\geq 2$. In group I, all patients had TIMI flow $>2(100 \%)$ (figure 1$)$. In group II, 41 patients had TIMI flow $>2(91.9 \%)$, while only 4 patients had TIMI flow $\geq 2(8.9 \%)$. There was statistically significant difference between the two groups ( $\mathrm{P}$ value $=0.041)$.

The lesion of the culprit vessel longer than $20 \mathrm{~mm}$ was found in 53 patients of the study population, and was shorter in 37 patients. In group I, longer lesion $<20 \mathrm{~mm}$ was found in all patients $(100 \%)$. In group II, 8 patients had the lesion longer than $20 \mathrm{~mm}(17.8 \%)$, while 37 patients had the lesion $>20 \mathrm{~mm}(82.2 \%)$ Table 4 . There was statistically significant difference between the two groups ( $P$ value $>0.001$ ).

Percutaneous coronary angioplasty (PTCA) was done in only 3 patients of the study population, and all of them were in the control group (6.7\%). The use of stents: stents were used in 87 patients of the study population table 4 . In group I: Bare Metal Stents (BMS) were used in 8 patients $(17.8 \%)$ and Drug Eluting Stents (DES) in 27 patients (82.2\%). In group II (BMS) were used in 10 patients $(22.2 \%)$ and DES in 32 patients $(71.1 \%)$. There was no statistically significant difference between the two groups ( $\mathrm{P}$ value $=0.167$ ).

\subsection{Oxidative Stress Markers}

Malondialdahyde (MDA); In group I, pt with coronary noreflow MDA level ranged from $2.8-4.5 \mathrm{nmol} / \mathrm{mL}$ with mean $3.9 \pm 1.5 \mathrm{nmol} / \mathrm{mL}$, while in group II control group, MDA level ranged from $1.1-2.1 \mathrm{nmol} / \mathrm{mL}$ with mean $1.55 \pm 0.4$ $\mathrm{nmol} / \mathrm{mL}$. There was statistically significant difference between the two groups ( $\mathrm{P}$ value $<0.004$ ) Figure 2 .

Superoxide dismutase (SOD); In group I, Superoxide dismutase level ranged from $58.1-65.2 \mathrm{U} / \mathrm{mL}$ with mean 61.9 $\pm 7.6 \mathrm{U} / \mathrm{mL}$, while in group II, Superoxide dismutase level ranged from 119.3-125.6 U/mL with mean $122.8 \pm 23.9$ x $10^{9} \mathrm{U} / \mathrm{mL}$. There was statistically significant difference between the two groups (P value $>0.002$ ). Figure 3

Reduced glutathione; In group I GSH ranged from 2224.1 (mg/dl of plasma) with mean $23.0 \pm 2.20$ while in group II, GSH ranged from $32-36.9$ (mg/dl of plasma) with mean $34.0 \pm 3.68$ Figure 4. There was statistically significant difference between the two groups ( $\mathrm{P}$ value $>0.01$ ).

Total antioxidant capacity (TAC); In group I, TAC mean was $2.15 \pm 0.27 \mathrm{mmol} / \mathrm{L}$ while in group II, TAC mean was $0.95 \pm 0.4 \mathrm{mmol} / \mathrm{L}$. There was statistically significant difference between the two groups ( $\mathrm{P}$ value $>0.004$ ) Figure 5 . 
Table 4. Coronary-angiographic findings during primary PCI of cases and control groups.

\begin{tabular}{|c|c|c|c|c|c|c|}
\hline \multirow{2}{*}{$\begin{array}{l}\text { Variables } \\
\text { Lesion Length }\end{array}$} & \multicolumn{2}{|c|}{ Case (no-reflow) $(n=45)$} & \multicolumn{2}{|c|}{ Control $(n=45)$} & \multirow{2}{*}{ Sig. test } & \multirow[t]{2}{*}{ P. } \\
\hline & & & & & & \\
\hline$<20$ & 0 & $0.0 \%$ & 37 & $82.2 \%$ & 62.830 & $<0.001 *$ \\
\hline$\geq 20$ & 45 & $100.0 \%$ & 8 & $17.8 \%$ & & \\
\hline \multicolumn{7}{|l|}{ MVD } \\
\hline No & 28 & $62.2 \%$ & 43 & $95.6 \%$ & \multirow[t]{2}{*}{15.011} & \multirow[t]{2}{*}{$<0.001 *$} \\
\hline Yes & 17 & $37.8 \%$ & 2 & $4.4 \%$ & & \\
\hline \multicolumn{7}{|c|}{ Lesion Localization } \\
\hline Proximal & 32 & $71.1 \%$ & 12 & $26.7 \%$ & \multirow{3}{*}{23.576} & \multirow{3}{*}{$<0.001 *$} \\
\hline Mid-segment & 13 & $28.9 \%$ & 20 & $44.4 \%$ & & \\
\hline Distal & 0 & $0.0 \%$ & 13 & $28.9 \%$ & & \\
\hline \multicolumn{7}{|l|}{ IRA } \\
\hline LAD & 34 & $75.6 \%$ & 21 & $46.7 \%$ & \multirow{3}{*}{11.132} & \multirow{3}{*}{$0.004 *$} \\
\hline LCX & 3 & $6.7 \%$ & 15 & $33.3 \%$ & & \\
\hline RCA & 8 & $17.8 \%$ & 9 & $20.0 \%$ & & \\
\hline \multicolumn{7}{|c|}{ TIMI Pre-Procedure } \\
\hline$>1$ & 0 & $0.0 \%$ & 4 & $8.9 \%$ & \multirow[t]{2}{*}{4.186} & \multirow[t]{2}{*}{$0.041 *$} \\
\hline$\leq 1$ & 45 & $100.0 \%$ & 41 & $91.1 \%$ & & \\
\hline \multicolumn{7}{|l|}{ Stent } \\
\hline PTCA only & 0 & $0.0 \%$ & 3 & $6.7 \%$ & \multirow{3}{*}{3.585} & \multirow{3}{*}{0.167} \\
\hline BMS & 8 & $17.8 \%$ & 10 & $22.2 \%$ & & \\
\hline DES & 37 & $82.2 \%$ & 32 & $71.1 \%$ & & \\
\hline \multicolumn{7}{|l|}{ TIMI Post } \\
\hline 0 & 37 & $82.2 \%$ & 0 & $0.0 \%$ & \multirow{4}{*}{90.000} & \multirow{4}{*}{$<0.001 *$} \\
\hline 1 & 8 & $17.8 \%$ & 0 & $0.0 \%$ & & \\
\hline 2 & 0 & $0.0 \%$ & 0 & $0.0 \%$ & & \\
\hline 3 & 0 & $0.0 \%$ & 45 & $100.0 \%$ & & \\
\hline
\end{tabular}

* Statistically Significant

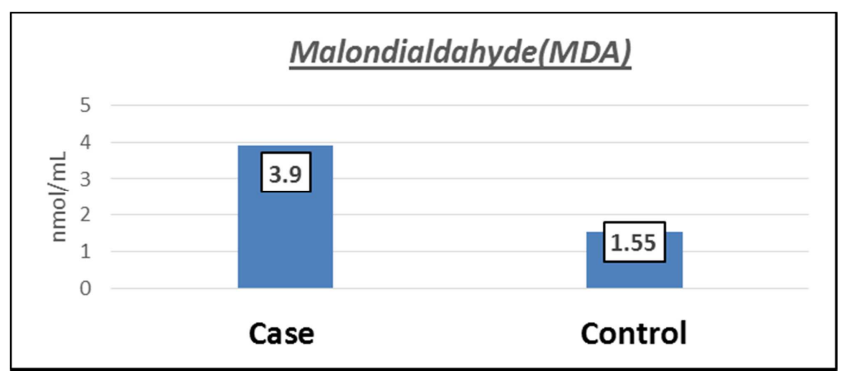

Figure 2. Malondialdahyde in both groups.

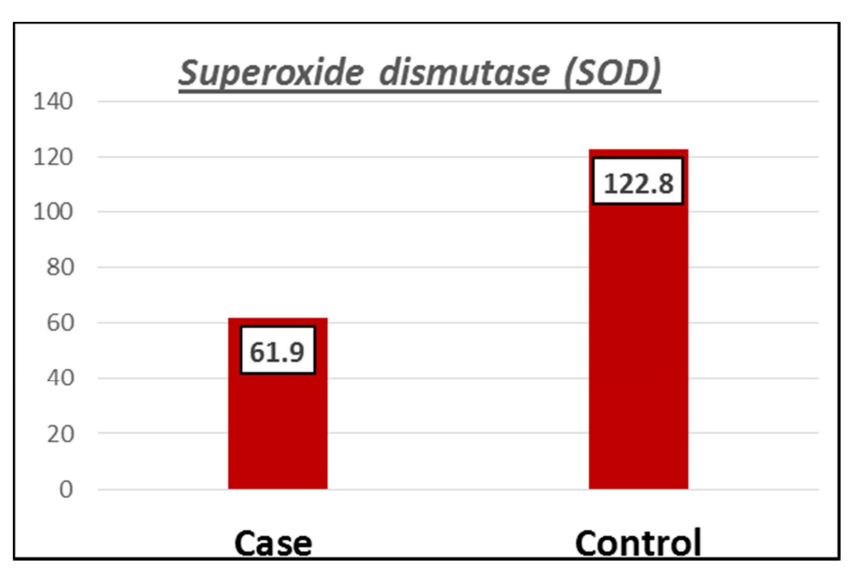

Figure 3. Superoxide dismutase (SOD level in both groups.

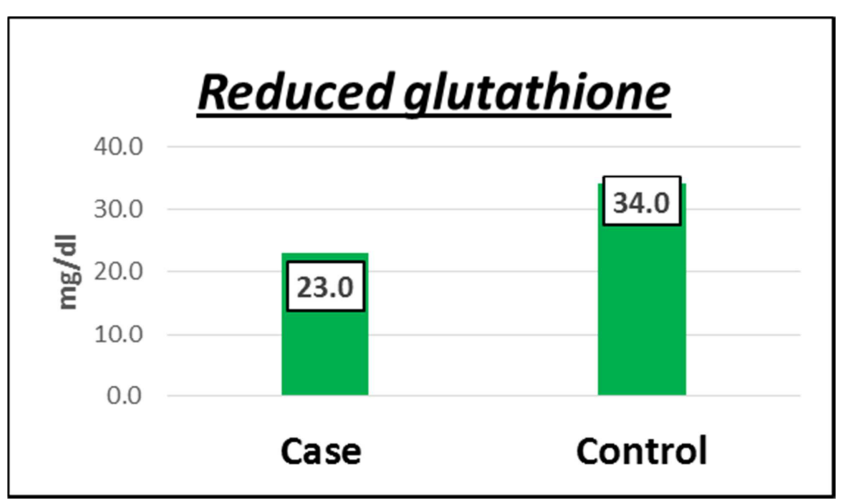

Figure 4. Reduced glutathione in both groups.

Table 5, showed that ejection fraction (EF) in 43 patients of the study population was $<40 \%$ upon admission, while was $>40 \%$ in the other 47 patients. In group 1, the EF was $>40 \%$ in 34 patients $(75.6 \%)$ while it was $<40 \%$ in 11 patients (24.4\%). In group 2, the EF was $>40 \%$ in 13 patients $(28.9 \%)$ while it was $<40 \%$ in 32 patients $(71.1 \%)$. There was statistically significant difference between the two groups (LV EF $<40 \%$, $\mathrm{P}$ value $<0.001$ ). 
Table 5. Echocardiographic findings of cases and control groups.

\begin{tabular}{|c|c|c|c|c|c|c|}
\hline \multirow[b]{2}{*}{ Ejection Fraction } & \multicolumn{2}{|c|}{ Case (no-reflow) $(n=45)$} & \multicolumn{2}{|c|}{ Control $(n=45)$} & \multirow[t]{2}{*}{ Sig. test } & \multirow[t]{2}{*}{$\mathbf{P}$. } \\
\hline & & & & & & \\
\hline$>40$ & 11 & $24.4 \%$ & 32 & $71.1 \%$ & 19.639 & $<0.001 *$ \\
\hline$<40$ & 34 & $75.6 \%$ & 13 & $28.9 \%$ & & \\
\hline
\end{tabular}

* Statistically Significant

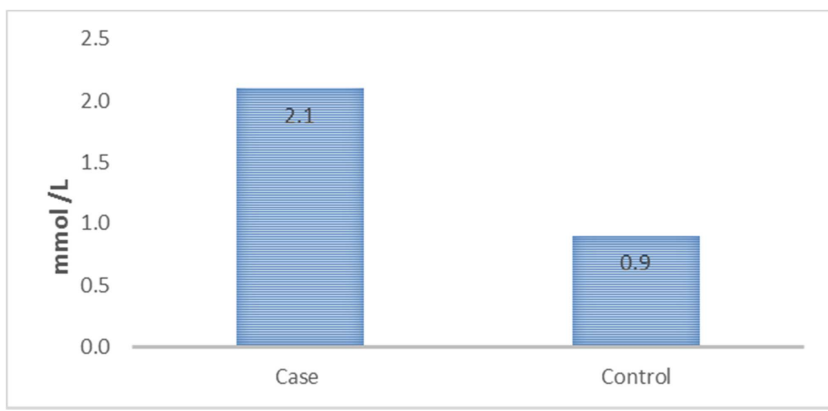

Figure 5. Total antioxidant capacity in both groups.

\section{Discussion}

AS regard Age \& Sex; in our study there was statistically significant difference between two groups as the majority of no reflow group were males $(91.1 \%$ vs $60 \%$, $\mathrm{P}$ value $>0.001)$ compared to control group, As regard Age there was statistically significant difference between two groups as the majority of no reflow group were found aged $<60$ years $(95.6 \%$ vs $17.8 \%$, $\mathrm{P}$ value 0.001$)$.

As regarding 2017 ESC guidelines ischemic heart disease develops on average 7-10 years later in women compared with men, MI remains a leading cause of death in women. Acute coronary syndrome (ACS) occurs three to four times more often in men than in women below the age of 60 years, but after the age of 75, women represent the majority of patients [15]. Women tend to present more often with atypical symptoms, up to $30 \%$ in some registries [3], and tend to present later than men [16].

In the study conducted with Sharif et al. [17] there was no significant difference between age and sex in relation to TIMI flow grade in patients presented with STEMI undergoing Primary PCI.

As regard DM \& Hypertension: There was statistically significant difference between 2 groups as the majority of no reflow group were diabetic $(84.4 \%$ vs $53.3 \%$, $\mathrm{P}$ value $=0.001)$, as regard hypertension there was statistically significant difference between 2 groups as the majority of no reflow group were hypertensive $(86.7 \%$ vs $46.7 \%$, $\mathrm{P}$ value $>0.001)$ as compared with control group.

As regard dyslipidemia \&smoking and family history of coronary artery disease: There was no statistically difference between 2 groups.

Similarly in the study conducted by Han et al.[18] which was conducted on 324 patients presented with STEMI that aimed to assess novel predictors of infarct-related artery patency for ST-segment elevation myocardial infarction that showed no significant difference between dyslipidemia and history of coronary artery diseases in patients presented with
STEMI undergoing primary PCI.

In contrast a prospective study was conducted by Rafizadeh et al. [19] on 880 patients with STEMI patients undergoing primary PCI to predict pre-interventional coronary artery patency which showed a significant difference between dyslipidemia and infract related coronary artery patency $(\mathrm{P}$ value $=0.049)$.

Similarly in the study conducted by Wang et al.[20] on 140 patients presented with STEMI undergoing primary PCI to assess effect of TIMI coronary flow in the culprit coronary artery on myocardial infarct and microvascular obstruction (MVO) size, there was no significant difference between smoking and pre-interventional TIMI flow.

As regard systolic blood pressure: there was statistically difference between 2 groups as the majority of no reflow group their systolic blood pressure less than $90 \mathrm{~mm} \mathrm{Hg}(62.2 \%$ vs $8.9 \%$, $\mathrm{P}$ value $<0.001$ ) as compared to control group.

As regard heart rate: there was no statistically difference between 2 groups ( $\mathrm{P}$ value $=0.438)$.

As regard random blood glucose: there was statistically difference between 2 groups as the majority of no reflow group their random blood glucose $<300 \mathrm{mg} / \mathrm{dl}(84.4 \%$ vs $31.1 \%$, $\mathrm{P}$ value $>0.001$ ) as compared to control group.

As regard KILLIP class: there was statistically difference between 2 groups as the majority of no reflow group their KILLIP class $\geq 3(62.2 \%$ vs $8.9 \%, P$ value $>0.001)$ as compared to control group.

Killip class $>2$ suggests that evidence of heart failure (HF) has been found. The correlation between HF and no reflow is a complex involving neurohumoral activation that leads to imbalance between nitric oxide (NO) and reactive oxygen species (ROS). Reduced bioavailability of NO and abundant formation of ROS within vascular wall play an important role in endothelial dysfunction which is the basic of pre-existing microvascular dysfunction mechanism [18].

A retrospective study was conducted by Han et al.[18] on 324 patients to assess predictors of infarct-related artery patency for ST-segment elevation myocardial infarction showed significant difference between the study groups as regarding KILLIP class ( $\mathrm{P}$ value=0.031).

As regard location of infarction: Patients of the no reflow group presented mainly with anterior STEMI (71.1\% vs $26.7 \%,<0.001)$.

As regard total ischemia time: Patients of the no reflow group presented mainly with ischemia time $<6 \mathrm{hrs}(100 \%$ vs $33.3 \%, \mathrm{p}>0.001)$.

In the study conducted by Wang et al., [21] no reflow cases were older and had higher heart rates than patients without No-reflow. They had a history of hypertension, diabetes and smoking. They presented with Killip class $>1$ on 
admission. Patients in the No-reflow group also had a longer total ischemia time. On the other hand, gender, hyperlipidemia, family history of coronary artery disease and systolic blood pressure significantly associated with no reflow.

In the study by Schram et al, patients in the no-reflow group had a significantly higher prevalence of diabetes mellitus and hypertension and Admission glucose $(125.6 \pm 71.3 \mathrm{mg} / \mathrm{dL})$ were significantly higher in the noreflow group. Between the two groups, there were no significant differences of age, sex distribution, dyslipidemia, ischemic time and infarct location [2].

Sabin Padmajan et al. in their study over 181 patients who underwent primary PCI, no reflow patients were $>60$ years. Reperfusion time was $>6$ h, Killip class was III/IV. On the other hand, there were no significant differences between the reflow group and the no-reflow group in terms of gender, hypertension, diabetes mellitus, hypercholesterolemia, smoking status, blood pressure (both systolic and diastolic), family history of coronary artery disease, serum creatinine and infarct localization ( $\mathrm{P}>0.05$ for all) [22].

In the no reflow group of the current study, LAD was predominantly the IRA $(75.6 \%$ vs $46.7 \%, \quad \mathrm{P}=0.004)$ compared to the control group. Also in the no reflow group, the IRA lesion was predominantly proximal $(71.1 \%$ vs $26.7 \%$, $\mathrm{P}>0.001)$ with longer lesion length $<20 \mathrm{~mm}(100 \%$ vs $17.8 \%$, $\mathrm{P}>0.001$ ).

Additionally, multi-vessel disease was more often found in the no reflow group $(37.8 \%$ vs $4.4 \%, \mathrm{P}>0.001)$.

There was also significant difference regarding preprocedural TIMI flow score between the two groups which found in no reflow group TIMI flow $\leq 1(100 \%$ vs $91.1 \%, \mathrm{P}$ value $=0.041)$. Also, there was no significant difference regarding revascularization method between the two groups either PTCA or Stent deployment.

In the study conducted by Celik et al., [23] TIMI flow 0-1 pre-PCI was more often present in the no-reflow group. Also, a larger infarct related vessel (IRV) diameter, longer infarct related lesion (IRL) length and were significantly higher in the no-reflow group. On the other hand, there was no significant difference in the presence of multivessel disease, and direct stenting between the two groups. Stents were significantly more used in the control group.

Wang et al. in their study, showed that no reflow was associated with pre-procedural TIMI flow, stent length, multivessel disease, balloon angioplasty with stenting. The LAD was predominantly the IRA in the no reflow patients [20].

In the study by Patterson et al., patients in the no reflow group had higher frequency of LAD as a culprit artery, proximal lesion localization, low pre-procedural TIMI flow, high grade thrombus burden and longer stent length. However, no reflow wasn't significantly associated with multivessel disease [24].

In contrast, a study conducted by Schaaf et al. [25] to assess Pre-PCI angiographic TIMI flow in the culprit coronary artery influences infarct size and microvascular obstruction in STEMI patients showed no significant difference between pre-interventional and post-interventional TIMI flow.

Lesion length and reference lumen diameter were found to be associated with no reflow. There are several aspects that may explain these results:

First, large vessels are able to accommodate large amounts of plaque lipid or thrombus. The larger the lesioned vessels are directly proportional to the slower the flow velocity, and the longer the target lesion reflects the larger amount of thrombus and plaque burden.

Left ventricular ejection fraction (LVEF) was statistically significant lower in the no-reflow group before primary PCI than the control group (LVEF $>40 \%, 75.6 \%$ vs $28.9 \%$, $\mathrm{P}>0.001$ ).

This came in agreement with the studies conducted by Gjin Ndrepepa et al. [5], Schram et al.[2]

Lipid peroxidation is a part of normal metabolism. Increased lipid peroxidation is thought to be a consequence of oxidative stress which occurs when the dynamic balance between oxidant and antioxidant mechanism is impaired [26]. We observed increased concentrations of Malondialdehide (MDA) in the circulation of patients with no-reflow indicating increased lipid peroxidation which could be attributed to a deficiency of antioxidant defense mechanism. In group I, pt with coronary no-reflow MDA level was (3.9 vs $1.1 \mathrm{nmol} / \mathrm{mL}$ ) for the control group, There was statistically significant difference between the two groups (P value $<0.004)$. This came in agreement with the study conducted by Holmes et al., [27] who studied the relation between Malondialdehyde and Low Density Lipoprotein as Oxidative-Stress Marker in Vasospastic Angina Patients and concluded that serum MDA, LDL may be a useful biomarker of oxidative stress and have additional value for the diagnosing the effect of oxidative stress in patients with vasospastic angina.

\section{Conclusion}

The occurrence of no-reflow phenomenon after primary PCI can be predicted using the oxidative stress markers such as reduction of SOD, reduced GSH and TAC levels with elevation of MDA level.

\section{References}

[1] Morishima I, Sone T, Okumura K, Tsuboi H, Kondo J, Mukawa $\mathrm{H}$, et al. Angiographic no-reflow phenomenon as a predictor of adverse long-term outcome in patients treated with percutaneous transluminal coronary angioplasty for first acute myocardial infarction. J Am Coll Cardiol. 2000; 36 (4): $1202-9$.

[2] Schram HCF, Hemradj V V., Hermanides RS, Kedhi E, Ottervanger JP. Coronary artery ectasia, an independent predictor of no-reflow after primary PCI for ST-elevation myocardial infarction. Int J Cardiol. 2018; (2017): \#pagerange\#. 
[3] Esteves Filho A, Garcia DP, Martinez Filho EE. The no reflow phenomenon in the coronary arteries. Arq Bras Cardiol. 1999 Jan; 72 (1): 99-108.

[4] Kloner RA. No-reflow phenomenon: maintaining vascular integrity. J Cardiovasc Pharmacol Ther 2011; 16: 244e50.

[5] Ndrepepa G, Tiroch K, Fusaro M, Keta D, Seyfarth M, Byrne RA, et al. 5-Year prognostic value of no-reflow phenomenon after percutaneous coronary intervention in patients with acute myocardial infarction. J Am Coll Cardiol 2010; 55: 2383e9.

[6] Niccoli G, Burzotta F, Galiuto L, Crea F. Myocardial noreflow in humans. J Am Coll Cardiol 2009; 54: $281 \mathrm{e} 92$.

[7] Fearon IM, Faux SP. Oxidative stress and cardiovascular disease: novel tools give (free) radical insight. J Mol Cell Cardiol 2009; 47: 372e81.

[8] Aviram M, Rosenblat M, Bisgaier CL, Newton RS, PrimoParmo SL, La Du BN. Paraoxonase inhibits high-density lipoprotein oxidation and preserves its functions. A possible peroxidative role for paraoxonase. J Clin Invest 1998; 101: $1581 \mathrm{e} 90$.

[9] Hsu CH, Chi BC, Liu MY, Li JH, Chen CJ, Chen RY. Phosphine-induced oxidative damage in rats: role of glutathione. Toxicology2002; 179: 1-8.

[10] Yeum KJ, Russell RM, Krinsky NI, Aldini G. Biomarkers of antioxidant capacity in the hydrophilic and lipophilic compartments of human plasma. Arch Biochem Biophys 2004; 430: 97-103.

[11] Erel O. A new automated colorimetric method for measuring totaloxidant status. Clin Biochem 2005; 38: 1103-11.

[12] Kampa M, Nistikaki A, Tsaousis V, Maliaraki N, Notas G, Castanas E. A new automated method for the determination of the Total Antioxidant Capacity (TAC) of human plasma, based on the crocin bleaching assay. BMC Clin Pathol 2002; 2: 3 .

[13] Erel O. A novel automated direct measurement method for total antioxidant capacity using a new generation, more stable ABTS radical cation. Clin Biochem 2004; 37: 277-85.

[14] Tatli E, Alicik G, Buturak A, Yilmaztepe M, Aktoz M. Arrhythmias following revascularization procedures in the course of acute myocardial infarction: are they indicators of reperfusion or ongoing ischemia? Scientific World Journal 2013; 160380 [PMID: 23431252 DOI: 10.1155/2013/160380].

[15] Faha Crcmdmf. The Netter Collection of Medical Illustrations - Cardiovascular System: Volume 8, 2e (Netter Green Book Collection). Saunders; 2014.

[16] Kumari SS, Menon VP. Changes in concentrations of lipid peroxides and activities of superoxide dismutase and catalase in isoproterenol induced myocardial infarction in rats. Ind J Exp Biol 1987; 25: 419-23.
[17] Sharif D, Abu-Salem M, Sharif-Rasslan A, Rosenschein U. Platelet counts on admission affect coronary flow, myocardial perfusion and left ventricular systolic function after primary percutaneous coronary intervention. Eur Hear journal Acute Cardiovasc care. 2017.

[18] Brunet J, Boily MJ, Cordeau S, Des Rosiers C. Effects of Nacetylcysteine in the rat heart reperfused after low-flow ischemia: evidence for a direct scavenging of hydroxyl radicals and a nitric oxide-dependent increase in coronary flow. Free Radic Biol Med 1995; 19: 627-638 [PMID: 8529922 DOI: 10.1016/0891-5849 (95)00077-B].

[19] Rafizadeh, Abdi S, O, Peighambari M, Basiri H, Bakhshandeh $\mathrm{H}$. Evaluation of the clinical and procedural predictive factors of no-reflow phenomenon following primary percutaneous coronary intervention. Res Cardiovasc Med. 2015.

[20] Wang JW, Zhou ZQ, Chen YD, Wang CH, Zhu XL. A risk score for No reflow in patients with ST-segment elevation myocardial infarction after primary percutaneous coronary intervention. Clin Cardiol. 2015.

[21] Han YL, Liu JN, Jing QM, Ma YY, Jiang TM, Pu K, et al. The efficacy and safety of pharmacoinvasive therapy with prourokinase for acute ST-segment elevation myocardial infarction patients with expected long percutaneous coronary intervention-related delay. Cardiovasc Ther. 2013.

[22] Sabin P, Koshy A, Gupta P, Heart - Indian, 2017 U. Predictors of no-reflow during primary angioplasty for acute myocardial infarction, from Medical College Hospital, Trivandrum. 2017 Apr; 69 Suppl 1 (Suppl 1): S34-S45.

[23] Celik T, Balta S, Mikhailidis DP, Ozturk C, Aydin I, Tok D, et al. The relation between no-reflow phenomenon and complete blood count parameters. Angiology. 2017.

[24] Patterson MRGSC. Netter's Cardiology 2nd Edition. second. 2010.

[25] Schaaf MJ, Mewton N, Rioufol G, Angoulvant D, Cayla G, Delarche N, et al. Pre-PCI angiographic TIMI flow in the culprit coronary artery influences infarct size and microvascular obstruction in STEMI patients. J Cardiol. 2016.

[26] Meier P, Gloekler S, Zbinden R, Beckh S, de Marchi SF, Zbinden S, et al. Beneficial effect of recruitable collaterals: a 10 -year follow-up study in patients with stable coronary artery disease undergoing quantitative collateral measurements. Circulation. 2007; 116 (9): 975-83.

[27] Holmes Jr DR, Bates ER, Kleiman NS, Sadowski Z, Horgan $\mathrm{JH}$, Morris DC, et al. Contemporary reperfusion therapy for cardiogenic shock: the GUSTO-1 trial experience. J Am Coll Cardiol 1995; 26: 668-74. 